\title{
REPRODUCTIVE PERFORMANCE OF WISTAR RATS
}

\author{
OHI, M.; DALSENTER, P.R. \\ Departmento de Farmacologia - UFPR, Curitiba/PR, Brazil, E.mail: masahiko@bio.ufpr.br
}

The reproductive performance of rats (Rattus rattus norvegicus), the main animal model for the research in reproductive toxicology, has been evaluated to compare with results from other laboratories and to subsidize the researches in the area. Fertility of the male rats was evaluated by mating adult males (200-250 g) in the proportion of one for two females. The regularity of the estrous cycle of adult female rats (190-250 g) was investigated by microscopic analysis of the vaginal material wash with physiologic solution. Reproductive indices and other values were attained by means of the mating of potentially fertile males with females diplaying normal cycle estrous. During the whole experiment the animals were maintained in acclimatized room provided with clear/dark cycles of 12 hours. Regularity of the estrous cycle was verified in $68,2 \%$ of the females $(\mathrm{N}=132)$. Reproductive index: mating of the males: $55,0 \%(\mathrm{~N}=20)$; fertility of the females: $71,6 \%(\mathrm{~N}=88)$; gestation: $100,0 \%(\mathrm{~N}=13)$; birth index: $96,4 \%(\mathrm{~N}=13)$; viability in the seventh day: $98,5(\mathrm{~N}=137)$ and in weaning: $85,7 \%(\mathrm{~N}=84)$; embryo resorption: $8,77 \%(\mathrm{~N}=150)$. Other values: the offspring relationship sex: 0,9 males for 1 female $(N=137)$; period (days) of gestation: 21,8 $(0,75(N=13)$; litter size: 10,5 (2,56 ( $\mathrm{N}=13)$; average of implants: $11,5(1,50(\mathrm{~N}=13)$. The body development $(\mathrm{g})$ of the females $(\mathrm{N}=13)$ during the experiment was the following: initial: 205 ( 16,2 ; in the mating: $246(15,7$; in weaning: $281(21,3$; in the sacrifice: $257(18,6$. The body development $(\mathrm{g})$ of the offspring in the lactation period (days) was: $1(5,96(0,65 ; \mathrm{N}=132) ; 7(14,2(1,67 ; \mathrm{N}=106) ; 14(24,9(2,89$; $\mathrm{N}=92) ; 21(37,4(4,80 ; \mathrm{N}=72)$. The weight $(\mathrm{g})$ of the uterus and ovary in the first estrous after delivery was $0,384(0,103(\mathrm{~N}=13)$ and $0,133(0,026$, respectively. These data, according to our experimental conditions, allow the conclusion that the reproductive performance of the animals used for research in the Biological Sciences Sector/UFPR agree, for this animal species, with the ones from another Brazilian research centers.

Key Words: Wistar rats; performance and reproductive indices. 